\title{
Lymphangiogenesis as a Prognostic Marker in Breast Cancer Using D2-40 as Lymphatic Endothelial Marker-A Preliminary Study*
}

\author{
Mumtaz A. Ansari ${ }^{1}$, Vaibhav Pandey ${ }^{1}$, Vivek Srivastava ${ }^{1}$, Mohan Kumar ${ }^{2}$, R. N. Mishra ${ }^{3}$, \\ Anand Kumar ${ }^{1 \#}$
}

${ }^{1}$ Department of General Surgery, Institute of Medical Sciences, Banaras Hindu University, Varanasi, India; ${ }^{2}$ Department of Pathology, Institute of Medical Sciences, Banaras Hindu University, Varanasi, India; ${ }^{3}$ Department of Biostatistics, Institute of Medical Sciences, Banaras Hindu University, Varanasi, India.

Email:mumtazbhu@gmail.com, sunny.ims@gmail.com,vivekims97@gmail.com,mohankumar@yahoo.co.in,

mishrarn@gmail.com, "profanandkumar52@gmail.com

Received August 23 ${ }^{\text {rd }}, 2012$; revised September $26^{\text {th }}, 2012$; accepted October $5^{\text {th }}, 2012$

\begin{abstract}
We studied tumour lymphangiogenesis and lymphatic invasion using D2-40 endothelial marker in 35 breast cancer patients treated by primary surgery and correlated it with various clinico-pathological prognostic parameters. Lymphangiogenesis was quantified using lymphatic micro vessel density (LMVD) by counting the immunostained lymphatic microvessels at 200×. The mean age was $45.97 \pm 12.09$ years (range $30-80$ years). LMVD ranged from 5/hpf to 56/hpf with a mean score of $13.4 \pm 10.8$ and median of 9 . The median value of 9 was taken to classify patients into a low or high LMVD. LMVD correlated significantly with tumour size $(p=0.003)$, histological grade $(p=0.046)$, lymph node status $(\mathrm{p}=0.030)$. There was no significant correlation of LMVD with stage, estrogen receptor, progesterone receptor or HER2/neu immunoreactivity. Lymphovascular invasion on D2-40 staining [LVI-D40] was found in 13 (37.1\%) cases compared to 6 cases $(17.1 \%)$ on H \& E staining showing a poor agreement $(\mathrm{k}=0.244)$. LVI correlated significantly with lymph node status $(\mathrm{p}=0.011)$. There was a strong association between tumour size $(\mathrm{p}=0.142)$, histological grade $(\mathrm{p}=$ 0.066 ) though the correlation was not statistically significant. No correlation was found with stage, estrogen receptor, progesterone receptor or HER2/neu immunoreactivity. The mean LMVD in LVI positive patients was higher (22.85 \pm $13.29)$ as compared to LVI negative patients $(7.95 \pm 2.05)$ and this was statistically significant $(p=0.001)$. Increased D2-40 detected LMVD and LVI correlated with poor prognostic parameters.
\end{abstract}

Keywords: Breast Cancer; Lymphangiogenesis, D2-40; Prognosis

\section{Introduction}

Breast cancer is the commonest cancer affecting women worldwide. Currently its management involves the assessment of various predictive and prognostic markers. Lymph node (LN) status is the most important independent clinical prognostic factor for patients with breast cancer [1]. This important prognostic benefit is not found in node negative patients and hence there is a need of a marker which can predict the subset of patients who will develop lymph node involvement eventually and can also serve as a prognostic marker. Lymphangiogenesis refers to the development and proliferation of new lymphatics from host vessels. Quantification of lymphangiogenesis has been done by measuring the lymphatic microvessel

\footnotetext{
"Conflict of interest: the authors of this article declare no conflict of interest.

${ }^{\#}$ Corresponding author.
}

density (LMVD). Changes in environment and composition of lymphangiogenic extracellular matrix, which is actively involved in tumor cell chemotaxis, may affect the function of both preexisting and newly formed lymphatics, promoting tumor cell invasion and dissemination. It has also been proposed that entry of cancer cells into the lymphatic vasculature might be facilitated by the higher permeability of proliferated lymphatic vessel and this enhances lymphatic metastasis assuming that tumor cells are passively taken up by lymphatic vessels along with the protein-rich interstitial fluid.

Lymphangiogenesis and invasion of lymphatics by tumour cells can serve as a surrogate marker in such situations. Though recent research on angiogenesis in cancer kinetics has been widely studied, yet lymphangiogenesis and the process of lymphatic invasion followed by metastasis to regional lymph nodes remains 
poorly understood. This lacuna in knowledge is primarily because of lack of lymphatic endothelial markers that can specifically identify the lymphatic endothelium.

Studies have demonstrated the clinical usefulness of quantifying lymphangiogenesis by lymphatic microvessel density (LMVD) estimation in head \& neck and lung cancer using specific markers for lymphatic vascular endothelium [2,3]. Since then, many studies have evaluated the LMVD as a prognostic factor for breast cancer $[4,5]$. LMVD was found to significantly correlate with the presence of regional or distant metastases and further shown to have a prognostic significance for breast cancer. However there was failure of consistent correlation between the LMVD and clinical outcome in breast cancer [5]. Controversies also exists about the role of peritumoural versus intratumoural lymphatics, site of lymphangiogenesis and the significance of mere lymphatic proliferation to qualify as an indicator for future nodal involvement. The lack of sensitivity of detecting only lymphatic endothelium was the major draw backs in these studies.

The monoclonal antibody D2-40 is shown in breast and tonsillar tissue to selectively detect lymphatic vessels in sections of both frozen and formalin fixed paraffin embedded normal and neoplastic tissues [5]. The methodology adopted to study lymphangiogenesis has been to find out the LMVD and the presence of tumour emboli within peritumoural endothelial lined spaces, defined as lymphovascular invasion (LVI). The identification of LMVD and LVI may permit the determination of patients at increased risk for axillary involvement and metastasis and thus can serve as a prognostic marker in node negative breast cancers.

The aim of this study was to determine the LMVD and LVI using D2-40 and their interrelationship with established prognostic markers in breast cancer.

\section{Material and Methods}

A prospective study was conducted in a single surgical unit of a University hospital from March 2008 to February 2010. All patients of carcinoma breast admitted in the unit during study period for surgery as primary modality of treatment were included in study. The patients who received any prior treatment like, lumpectomy, neo-adjuvant chemotherapy or Radiotherapy were excluded. Patients who had any kind of breast surgery in past or who did not give consent were also excluded. The diagnosis was made by FNAC from breast lump after detailed clinical evaluation. Patients underwent modified radical mastectomy followed by detailed histological examination of specimen, receptor status evaluation and LMVD and LVI estimation using D2-40 as primary antibody. Adjuvant treatment in the form of chemotherapy and/or radiotherapy was given if indicated to complete the therapy. After completion of treatment patients were kept on three monthly follow up for two year and thereafter every six months. Metastasis is recorded during the follow up period.

Histological examination was done after hematoxylin and eosin (H \& E) staining and tumour was graded according to the Bloom Richardson Grading system. The presence of tumour emboli within peritumoural endothelial lined spaces, stained with $\mathrm{H} \& \mathrm{E}$ was defined as positive LVI. Hormone receptor status was determined by immunohistochemistry.

Blocks of the viable and tumour representative area were selected for LMVD and LVI estimation using immunohistochemistry. Tissue sections $(4 \mu \mathrm{m}$ thick) were dewaxed and antigen retrieval was done by incubation in microwave using citrate buffer $(\mathrm{Ph} \mathrm{6})$ at $95^{\circ} \mathrm{C}$ and $97^{\circ} \mathrm{C}$ for 10 minutes each. Then the sections were incubated in $3 \% \mathrm{H}_{2} \mathrm{O}_{2}$ solution for blocking endogenous peroxidase. Following this the tissue sections were incubated in primary antibody D2-40 [1:50 dilution] (DAKO corporation, Denmark) solution in humid chamber for 30 minutes at room temperature. After washing, the sections were treated with biotinylated secondary antibody followed by avidin coupled to biotinylated peroxidase at room temperature [LSAB kit] (DAKO corporation, Denmark). Immunohistochemical reaction were developed with diamino benzidine dihydrochloride (DAB) chromogen peroxidise substrate and counterstained with Haematoxylin and Eosin. A block of archival tonsillar tissue served as positive control [6]. For negative control, a slide was prepared from the same tissue block and a preimmune serum was used instead of the primary antibody.

\section{Lymphatic Microvessel Density (LMVD) and Lymphatic Vascular Invasion (LVI) Assessement}

The determination of microvessels was done using criteria described by Weidner et al, 1991 [7]. Lymphatic vessels were defined as the vessels which have endothelium with immune-positivity to D2-40 and had a lumen. The sections were initially scanned at low magnification $(40 \times)$, thereby finding the areas with the highest number of lymphatic microvessels (the hot spots). LMVD was then determined by counting all D2-40 immunostained vessels at high magnification $(200 \times)$ in three hot spots (Figures 1 and 2). Determination of staining was strictly confined to hot spots. Microvessel counts were done by two independent observers, naive to the patient's pathologic and clinical status. The mean value of microvessel densities observed by both investigators in each patient was entered into further calculations. In case of inter-observer difference of more than $30 \%$ in microvessel count, the respective slides were reinvestigated by both 


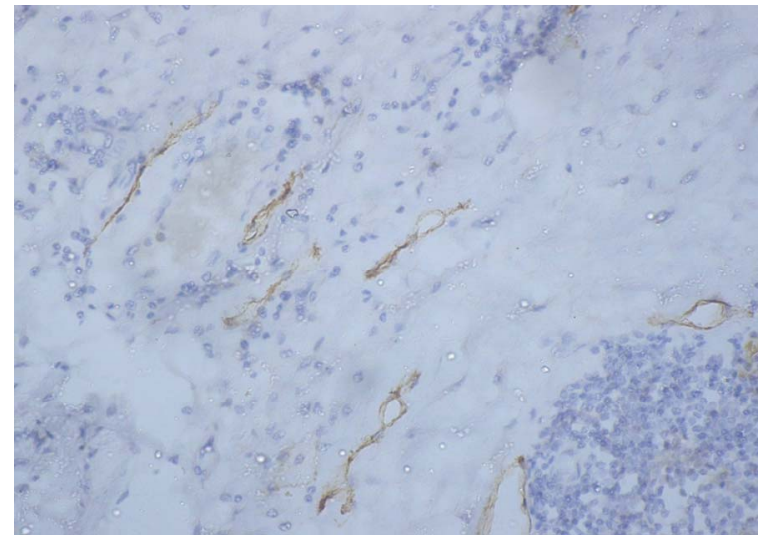

Figure 1. Invasive breast carcinoma showing positive immunostaining of lymph vessels with D2-40 (100×). No tumour embolus seen.

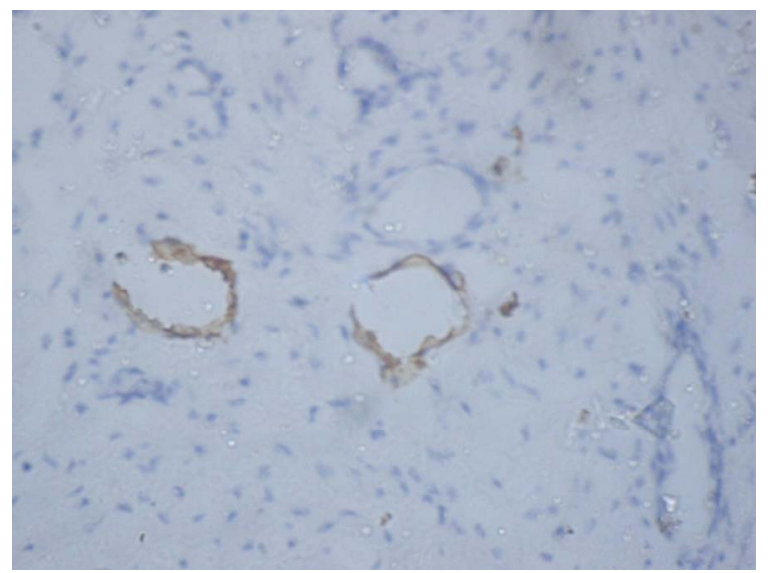

Figure 2. Lymphovascular bundle stained with D2-40 with positive staining of lymphatic endothelium $(200 \times)$. The adjacent blood vessel endothelium is not stained.

observers using a discussion microscope (evident in $<5 \%$ of cases).

LVI was recognized as tumour cell nests floating within empty spaces, which were surrounded by thin, spindle shaped endothelial cells. A lymph vessel that showed positive staining of the endothelium for D2-40 which surrounded the tumour cell nests was diagnosed as positive for LVI (Figure 3). The evaluation of LVI by the hematoxilin and eosin stained sections was also documented for the study. The D2-40-stained slide was assessed for lymph vessel invasion without knowledge of the LVI status based on the hematoxilin and eosin staining. Interpretation of immunohistochemical results were made without knowledge of clinical stage or the status of other prognostic variables. LMVD and LVI were correlated with other prognostic parameters besides each other.

Statistical analysis was done using SPSS software version 14. The mean difference between two groups was

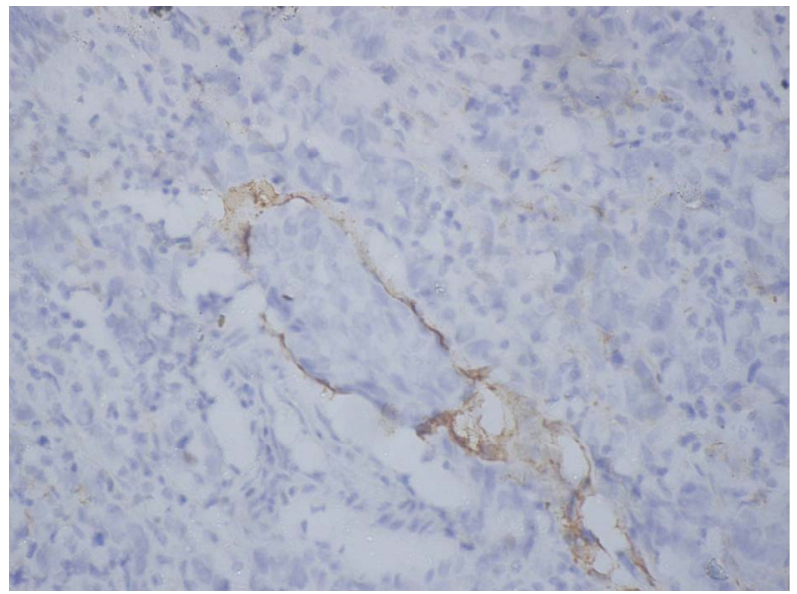

Figure 3. Positive staining of lymphatic endothelium with D2-40 outlining a tumour embolus in the lumen $(200 \times)$. This tumour embolus could not be visualized on the $H$ \& $E$ section as it completely obliterates the lumen.

calculated by student $t$ test for unpaired data and chi squared test was done for significance of difference among two proportions. Correlation among qualitative data was done by Pearson correlation. Kaplan Meier curve was used to visualize the survival rate and log rank test was performed to compare the survival rates. The level significance was considered at $5 \%$ cut-off point. The significance level more than $5 \%$ written as $p>0.05$ was taken as statistically insignificant.

\section{Results}

A total of thirty five female patients entered into study during the study period. The mean age was $45.97 \pm 12.09$ years (range 30 - 80 years). Clinicopathologic parameters of these patients are given in Table 1.

Lymphangiogenesis was quantified by microvessel density, using D2-40 as markers for lymphatic endothelium. In our study LMVD ranged from 5/hpf to 56/hpf with mean LMVD of $13.4 \pm 10.8$ and median LMVD of 9. The median value of 9 was taken to classify patients into a low or high LMVD score as a reference value. The cases thus were divided based on low or high LMVD and was compared with other prognostic markers viz. tumour size, stage, number of lymph node involvement, histological grade and receptor status. The results are shown in Table 2. LMVD correlated significantly with tumour size $(p=0.003)$, histological grade $(p=0.046)$, lymph node status $(p=0.030)$. There was no significant correlation of LMVD with stage, estrogen receptor, progesterone receptor or HER2/neu immunoreactivity.

Lymphovascular invasion on D2-40 staining [LVID40] was found in 13 (37.1\%) cases compared to 6 cases (17.1\%) on $\mathrm{H} \& \mathrm{E}$ staining. Out of 13 LVI positive cases, 9 cases were positive for LVI on D2-40 only and 
Table 1. Clinicopathologic characteristics of thirty five patients.

\begin{tabular}{|c|c|}
\hline Characteristics & \\
\hline \multicolumn{2}{|l|}{ Age } \\
\hline Mean (years) & $45.97 \pm 12.09$ \\
\hline Range (years) & $30-80$ \\
\hline \multicolumn{2}{|c|}{ Menstrual status, No (\%) } \\
\hline Premenopausal & $18(51.4)$ \\
\hline Postmenopausal & $17(48.6)$ \\
\hline \multicolumn{2}{|l|}{ Tumour size $(\mathrm{cm})$} \\
\hline T 1 & - \\
\hline Т 2 & $18(51.4)$ \\
\hline T 3 & $17(48.6)$ \\
\hline Clinical N-status & $11(31.43)$ \\
\hline $\mathrm{N}_{0}$ & $14(40.0)$ \\
\hline $\mathrm{N}_{1}$ & $10(28.57)$ \\
\hline $\mathrm{N}_{2 \mathrm{a}}$ & - \\
\hline $\mathrm{N}_{2 b}$ & 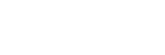 \\
\hline \multicolumn{2}{|l|}{ Stage } \\
\hline IIa & $16(45.7)$ \\
\hline IIb & $13(37.1)$ \\
\hline IIIa & \\
\hline \multicolumn{2}{|l|}{ Grade } \\
\hline I & $1(2.9)$ \\
\hline II & $10(28.6)$ \\
\hline III & $24(68.6)$ \\
\hline \multicolumn{2}{|c|}{ No. of involved Lymph Nodes } \\
\hline 0 & $14(40.0)$ \\
\hline $1-3$ & $7(20.0)$ \\
\hline $4-9$ & $5(14.3)$ \\
\hline$>10$ & $9(25.7)$ \\
\hline \multicolumn{2}{|l|}{ ER } \\
\hline Positive & $3(13.63)$ \\
\hline Negative & $19(86.36)$ \\
\hline \multicolumn{2}{|l|}{ PR } \\
\hline Positive & $3(13.63)$ \\
\hline Negative & $19(86.36)$ \\
\hline \multicolumn{2}{|l|}{ HER2/neu } \\
\hline Positive & $9(40.90)$ \\
\hline Negative & $13(59.09)$ \\
\hline
\end{tabular}

negative on $\mathrm{H} \& \mathrm{E}$ staining while only 2 cases were positive for LVI on $\mathrm{H} \& \mathrm{E}$ but negative on D2-40 staining. Four cases were positive on both D2-40 and H \& E. The kappa score obtained in our study showed poor agreement regarding the LVI when comparing D2-40 immunostained and $\mathrm{H} \& \mathrm{E}$ stained sections $(\mathrm{k}=0.244)$. Table 3 shows comparision of LVI on D2-40 staining correlated with other prognostic parameters. LVI correlated signifycantly with lymph node status $(p=0.011)$. There was a strong association between tumour size $(\mathrm{p}=0.142)$, histological grade $(\mathrm{p}=0.066)$ though the correlation was not statistically significant. No correlation was found with stage, estrogen receptor, progesterone receptor or HER2/neu immunoreactivity. The mean LMVD in LVI positive patients was higher $(22.85 \pm 13.29)$ as compared to LVI negative patients $(7.95 \pm 2.05)$ and this was statistically significant $(\mathrm{p}=0.001)$. Five out of thirty five patients developed metastasis during follow up, three in liver and two in spine. Four out of these five patients showed lympho vascular invasion while one was negative $(\mathrm{p}=0.032)$.

\section{Discussion}

Breast cancer is the second leading cause of cancer deaths among woman worldwide [8]. The concept of tumour induced lymphangiogenesis has been met with

Table 2. Comparison of LMVD with other prognostic parameters.

\begin{tabular}{|c|c|c|c|}
\hline & \multicolumn{2}{|c|}{ LMVD } & \multirow{3}{*}{ p-value } \\
\hline & $<9$ & $\geq 9$ & \\
\hline & No. $(\%)$ & No. (\%) & \\
\hline Mean tumour size & $4.20 \pm 1.61$ & $6.10 \pm 1.83$ & 0.003 \\
\hline Stage & & & 0.388 \\
\hline IIA & $4(26.7)$ & $2(10.0)$ & \\
\hline IIB & $7(46.7)$ & $9(45.0)$ & \\
\hline IIIA & $4(26.7)$ & $9(45.0)$ & \\
\hline Positive lymph node & & & 0.030 \\
\hline $1-3$ & $3(100)$ & $4(22.2)$ & \\
\hline $4-9$ & - & $5(27.8)$ & \\
\hline$>10$ & - & $9(50.0)$ & \\
\hline Grade & & & 0.046 \\
\hline I & $1(6.7)$ & - & \\
\hline II & $7(46.7)$ & $3(15.0)$ & \\
\hline III & $7(46.7)$ & $17(85.0)$ & \\
\hline ER & & & 0.907 \\
\hline Positive & $1(12.5)$ & $2(14.3)$ & \\
\hline Negative & $7(87.5)$ & $12(85.7)$ & \\
\hline PR & & & 0.907 \\
\hline Positive & $1(12.5)$ & $2(14.3)$ & \\
\hline Negative & $7(87.5)$ & $12(85.7)$ & \\
\hline HER & & & 0.512 \\
\hline Positive & $4(50.0)$ & $5(35.7)$ & \\
\hline Negative & $4(50.0)$ & $9(64.3)$ & \\
\hline
\end{tabular}


Table 3. Comparison of LVI with other prognostic parameters.

\begin{tabular}{|c|c|c|c|}
\hline & \multicolumn{2}{|c|}{ LVI D2-40 } & \multirow{3}{*}{ p-value } \\
\hline & Positive & Negative & \\
\hline & No. (\%) & No. (\%) & \\
\hline Mean Tumour Size & $5.92 \pm 1.55$ & $4.91 \pm 2.11$ & 0.142 \\
\hline Stage & & & 0.755 \\
\hline IIA & $2(15.4)$ & $4(18.2)$ & \\
\hline IIB & $7(53.8)$ & $9(40.9)$ & \\
\hline IIIA & $4(30.8)$ & $9(40.9)$ & \\
\hline Grade & & & 0.066 \\
\hline I & - & $1(4.5)$ & \\
\hline II & $1(7.7)$ & $9(40.9)$ & \\
\hline III & $12(92.3)$ & $12(54.5)$ & \\
\hline Positive lymph node & & & 0.011 \\
\hline $1-3$ & $1(8.3)$ & $6(66.7)$ & \\
\hline $4-9$ & $3(25.0)$ & $2(22.2)$ & \\
\hline$>10$ & $8(66.7)$ & $1(11.1)$ & \\
\hline ER & $1(9.1)$ & $2(18.2)$ & 0.534 \\
\hline Positive & $10(90.9)$ & $9(81.8)$ & \\
\hline Negative & & & \\
\hline PR & & & 0.534 \\
\hline Positive & $1(9.1)$ & $2(18.2)$ & \\
\hline Negative & $10(90.9)$ & $9(81.8)$ & \\
\hline HER & & & 0.665 \\
\hline Positive & $4(36.4)$ & $5(45.5)$ & \\
\hline Negative & $7(63.6)$ & $6(54.5)$ & \\
\hline Mean LMVD & $22.85 \pm 13.29$ & $7.95 \pm 2.05$ & 0.001 \\
\hline $\begin{array}{l}\text { Metastatic disease in } \\
\text { follow up }\end{array}$ & & & 0.032 \\
\hline Present & $4(30.8)$ & $1(4.5)$ & \\
\hline Absent & $9(69.2)$ & $21(95.5)$ & \\
\hline
\end{tabular}

skepticism in the past and some what neglected. It was considered that tumour associated lymphatics were pre existing and not newly formed [9]. Recent research has provided sufficient evidence that tumours are not only able to induce lymphangiogenesis but also that this leads to enhanced lymphatic metastasis [10-12]. Tumour lymphangiogenesis can be studied by various parameters like estimation of expression of VEGF family gene products by immunohistochemistry or quantitative RT-PCR and by lymphatic vessel density assessment using antibodies against proteins specifically expressed on lymphatic endothelium. Several lymphatic endothelial markers have been established recently like podoplanin, desmoplakin, Prox 1, receptors for VEGF-C and VEGF-D (VEGFR-3).
Podoplanin is a 38-KD surface glycoprotein expressed on lymphatic but not blood vascular endothelium has been demonstrated in the skin. Non specific staining of blood vessels has been reported [13]. Desmoplakin is a glycolprotein located exclusively to the intracellular junctions between the endothelial cells of lymphatic vessels [14]. Antibodies against desmoplakin have indicated specificity for lymphatic endothelium in human tongue but further studies are required to confirm the distinctive nature of desmoplakin staining in other tissue types. Prox-I is required for the regulation of lymphatic vascular development from pre-existing embryonic veins [15]. Antibodies against human Prox-1 to visualize lymphatic vessels in tumour sections have only been used in a limited number of studies. The vascular endothelial growth factor receptor 3 (VEGFR-3) is a tyrosine kinase that is predominantly expressed in Lymphatic endothelial cells in adult tissues, this marker is not reliable for discriminating between lymphatic and blood vascular endo thelium. Several studies have shown a worse prognosis for tumours with high lymphangiogenic activity $[16,17]$.

This was a preliminary study of lymphangiogenesis in 35 carcinoma breast patients using D2-40 endothelial marker for estimation of LMVD and determining its prognostic significance. A significant correlation between D2-40 detected LMVD with other unfavorable prognosis parameters was found. This was in consistence with studies done in malignant melanoma and bladder cancer where a significant correlation between LMVD with the occurrence of lymphatic metastases and survival was observed [16,17]. Tumour cells are carried to regional lymph nodes through the lymphatics as tumour emboli. Thus, the detection of peritumoural lymphatic vessels and its invasion (LVI) on histological sections can predict lymph node metastasis of the disease in clinically node negative patients and can serve as an important prognostic marker.

Histological sections are routinely studied on $\mathrm{H}$ \& E staining but it has a limitation of poor yield in lymphatic vessel visualization and detection of invasion. This non visualization of lymphatics is because of non staining of the lymphatic endothelial cells. Majority of tumour emboli completely obliterate the lumen of the lymphatics and they can not be differentiated from nests of tumour cells which are tumour retraction artifacts and isolated tumour aggregate due to tissue shrinkage during fixation in routine $\mathrm{H} \& \mathrm{E}$ staining. Contrary to this D2-40 selectively stains the lymphatic endothelium. This method is suitable for enhanced visualization of lymphatics as well as for accurate detection of LVI. In a study done by Kahn and Marks on 50 breast cancer cases, there was an increase of $18 \%$ in LVI detection on D2-40 when compared to H \& E staining [18]. Marinho et al. showed 
similar detection rates with $28.5 \%$ and $13.8 \%$ of LVI on D2-40 and $\mathrm{H} \& \mathrm{E}$ staining respectively [19]. Our result with D2-40 detected LVI was $37.1 \%$ compared to $17.1 \%$ by routine $\mathrm{H} \& \mathrm{E}$ staining. Thus there was a two fold increase of LVI detection rate using D2-40. The kappa score obtained in the study showed poor agreement between LVI detection on D2-40 immunostaining compared with $\mathrm{H} \& \mathrm{E}$ stained sections $(\mathrm{k}=0.244)$.

\subsection{Prognostic Parameter Correlation}

\subsubsection{Lymph Nodes}

It was emphasized that a correlation between LMVD and LVI coexists [20]. The study observed that mean LMVD in LVI positive and negative cases to be $12 \pm 4.2$ and 8.3 $\pm 4.2(\mathrm{p}=0.001)$. This significant association between LMVD and LVI could be explained through a lymphangiogenesis-induced increase of the "lymphatic window" providing tumour cells with more opportunities to enter into lymphatic vessels. The present study observed a similar result with mean LMVD in LVI positive cases of $22.85 \pm 13.29$ and in LVI negative cases of $7.95 \pm 2.05$ $(p \leq 0.001)$. This signifies that a higher mean LMVD to be present in LVI positive cases as compared to LVI negative cases. The result suggests that breast cancers with high peritumoural lymphangiogenesis as measured with LMVD more often invade these lymphatic vessels and have more chance of lymphatic metastasis as compared to patients with low LMVD. This is further supported with the observation that $85.7 \%$ patients with metastatic deposits in the axillary lymph nodes had LMVD score more than the median $(p=0.030)$. Further, a significant correlation between the total number of positive lymph nodes and LMVD $(r=0.863, p \leq 0.0001)$ also supports the statement. Similar results were obtained by Xie et al. on 102 patients of breast cancer where they found correlation of LMVD with the number of metastatic lymph node $(r=0.964, p<0.01)$ [21].

Tumor associated lymphatic vessels in breast cancer are considered as the main route of tumour cells to axillary lymph nodes and tumour cells exposed to more microvessels are more likely to spread to distant sites and to lymph nodes [22]. A significant correlation between the LVI positivity and metastatic lymph node status was observed in the study $(\mathrm{p}=0.011)$. LVI positivity increased from $7.1 \%$ in patients with no nodal metastasis to $88.8 \%$ in patients $>10$ nodes metastasis. The study suggests that LVI alone can predict metastatsis to axillary lymph node. Similar results were observed by Kahn et al. who demonstrated LVI positivity in $44 \%$ of node negative and in $86 \%$ of node positive breast cancer patients [18]. Gurleyik et al. in study of 81 patients showed that when axillary involvement progressed from negative to more than ten nodes, the rate of positive LVI increased from $14 \%$ to $100 \%$ [23].

\subsubsection{Tumour Size, Grade and Receptor Status}

Tumour size is another important prognostic marker in breast cancer and predictor of local recurrence, regional and/or systemic spread and overall survival [24,25]. A positive correlation between tumor size and LMVD was suggested $[4,26]$. Similar observations were found in the present study where the mean tumour size in patients with high LMVD was significantly more than those with low LMVD.

It is speculated that fast growing tumours produce more growth factors and offer a bigger clonal variety of tumour cells capable of involving lymphatic vessels compared with well differentiated slow growing tumours. Thus it is expected to have a high LMVD associated with low histologic differentiation grade. Schoppmann et al. and Kato et al. have shown a significant inverse correlation between grade of tumour with LMVD $[15,20]$. In the present study out of 20 patients with high LMVD, 85\% were Grade III and $15 \%$ grade II and while out of 15 low LMVD patients $46.7 \%$ were grade III, $46.7 \%$ grade II and $6.7 \%$ grade I $(p=0.046)$. An increasing trend of LVI positivity with increasing grade of tumour is suggested. None of the grade I tumour patients was LVI positive, compared to $50 \%$ LVI positivity in grade III tumour patients $(\mathrm{p}=0.066)$. Conversely, $92.3 \%$ of LVI positive cases were of histological grade III. Gurleyik et al. reported that LVI positivity increased up to $73 \%$ when the tumour grade was III, whereas there were no positive LVI in grade I tumours [23].

It is true that aggressive tumours have negative receptor status and this should reflect in terms of LMVD and LVI also, but studies have reported different results of receptor status with respect to LVI and LMVD and no unanimity exists in this regard, Gurleyik et al. reported positive LVI in $85 \%$ of estrogen receptor negative tumours $(\mathrm{p}<0.0001)$ and in $73 \%$ of progesterone receptor negative tumours $(\mathrm{p}=0.0004)$ [23]. However, Kato et al. found no significant correlation between ER/PR status and LMVD [15]. Schopmann et al. also reported positive LVI in only $28.2 \%$ patients out of 78 estrogen receptor negative patients $(p=0.961)$ [20]. Similar to these findings the present study found high LMVD in $36.8 \%$ ( $p=$ $0.907)$ and positive LVI in only $52.6 \%(\mathrm{p}=0.534)$ of estrogen and progesterone receptor negative tumours.

\subsubsection{Metastatic Potential}

The risk of developing lymph node metastasis increases significantly with the presence of lymphovascular invasion and hence it can be regarded as the predictor of nodal involvement and also the disease free survival. Schoppmann et al. [20] and Kato et al. [15] compared disease free survival with LMVD and failed to find a significant correlation. Schopmann et al. [20] found a significant difference in overall survival and disease free 
survival between patients with or without LVI both in univariate and multivariate survival analysis. In the present study five out of thirty five patients developed metastasis in follow up. Though a significant correlation between disease free survival and LMVD could not be drawn yet the mean LMVD in patients who developed metastasis was higher as compared to those who were free of metastasis $(21.6 \pm 12.66$ and $12.13 \pm 10.16$ respectively) $(p=0.071)$. On the other hand the LVI positivity was significantly higher in patients who developed metastasis $(p=0.018)$. The observation favors the statement that the patients with lymphatic embolization are more prone for metastatic disease.

\section{Conclusion}

The system of lymphangiogenesis represents a potential new target for cancer prognostication and development of anti-cancer strategies. Specific lymphatic endothelial markers, such as podoplanin, Prox-1, and LYVE-1, now provide sufficient tools to researchers to understand better, the concepts of tumour lymphangiogenesis. The data presented herein support the importance LVI and LMVD assessment using D2-40 in breast cancer patients for prognostic purpose. The higher positivity of LMVD and LVI correlated with other established prognostic measures like tumour size, lymph node metastasis, number of involved nodes, grade of tumour and tumour metastasis. This highlights the use of this novel marker in identification of patients who will have a poor prognosis even if they have early cancer without nodal involvement. LMVD and LVI assessment using D2-40 can be used as a single prognostic marker in primary breast cancer but needs multicentric study and a longer follow up for its validity which can probably establish the entity.

\section{Acknowledgements}

The authors acknowledge the contribution of Prof Saroj Gupta for her contribution in pathological examination.

\section{REFERENCES}

[1] E. R. Fisher, et al., "Pathologic findings from the $\mathrm{Na}-$ tional Surgical Adjuvant Breast Project (NSABP) Protocol B-17: Intraductal Carcinoma (Ductal Carcinoma in Situ)," Cancer, Vol. 75, No. 6, 1995, pp. 1310-1319. doi:10.1002/1097-0142(19950315)75:6<1310::AID-CNC R2820750613>3.0.CO;2-G

[2] P. A. Kyzas, et al., "Evidence for Lymphangiogenesis and Its Prognostic Implications in Head and Neck Squamous Cell Carcinoma," Journal of Pathology, Vol. 206, No. 2, 2005, pp. 170-177. doi: $10.1002 /$ path. 1776

[3] K. Schmid, et al., "Prognostic Value of Lymphatic and Blood Vessel Invasion in Neuroendocrine Tumours of the
Lung," The American Journal of Surgical Pathology, Vol. 29, 2005, pp. 324-328. doi:10.1097/01.pas.0000149706.74216.b6

[4] P. Bono, et al., "High LYVE-1-Positive Lymphatic Vessel Numbers Are Associated with Poor Outcome in Breast Cancer," Clinical Cancer Research, Vol. 10, No. 21, 2004, pp. 7144-7149. doi:10.1158/1078-0432.CCR-03-0826

[5] C. S. M. Williams, et al., "Absence of Lymphangiogenesis and Intratumoural Lymph Vessels in Human Metastatic Breast Cancer," The Journal of Pathology, Vol. 200, 2003, pp. 195-206. doi:10.1002/path.1343

[6] H. J. Kahn, et al., "Monoclonal Antibody D2-40, a New Marker of Lymphatic Endothelium, Reacts with Kaposi's Sarcoma and a Subset of Angiosarcomas," Modern Pathology, Vol. 15, No. 4, 2002, pp. 434-440. doi:10.1038/modpathol.3880543

[7] N. Weidner, et al., "Tumour Angiogenesis and Metastasis - Correlation in Invasive Breast Carcinoma," The New England Journal of Medicine, Vol. 324, No. 1, 1991, pp. 1-8. doi:10.1056/NEJM199101033240101

[8] R. G. C. Dumitrescu, "Understanding Breast Cancer Risk Where Do We Stand in 2005?" Journal of Cellular and Molecular Medicine, Vol. 9, No. 1, 2005, pp. 208-221. doi:10.1111/j.1582-4934.2005.tb00350.x

[9] A. J. Leu, et al., "Absence of Functional Lymphatics within a Murine Sarcoma: A Molecular and Functional Evaluation," Cancer Research, Vol. 60, No. 16, 2000, pp. 4324-4327.

[10] M. Skobe, et al., "Induction of Tumour Lymphangiogenesis by VEGF-C Promotes Breast Cancer Metastasis," Nature Medicine, Vol. 7, No. 16, 2001, pp. 192-198. doi:10.1038/84643

[11] S. J. Mandriota, et al., "Vascular Endothelial Growth Factor-C Mediated Lymphangiogenesis Promotes Tumour Metastasis," The EMBO Journal, Vol. 72, No. 20, 2001, pp. 672-682. doi:10.1093/emboj/20.4.672

[12] Y. He, et al., "Suppression of Tumour Lymphangiogenesis and Lymph Node Metastasis by Blocking Vascular Endothelial Growth Factor Receptor 3 Signaling," Journal of the National Cancer Institute, Vol. 94, No. 11, 2002, pp. 819-825. doi:10.1093/jnci/94.11.819

[13] S. Breiteneder-Geleff, et al., "Angiosarcomas Express Mixed Endothelial Phenotypes of Blood and Lymphatic Capillaries: Podoplanin as a Specific Marker for Lymphatic Endothelium," American Journal of Pathology, Vol. 154, 1999, pp. 385-394. doi:10.1016/S0002-9440(10)65285-6

[14] M. Schmelz and W. W. Franke, "Complexus Adhaerentes, a New Group of Desmoplakin-Containing Junctions in Endothelial Cells: The Syndesmos Connecting Retothelial Cells of Lymph Nodes," European Journal of Cell Biology, Vol. 61, No. 2, 2005, pp. 274-289.

[15] J. T. Wigle and G. Oliver, "Prox1 Function Is Required for the Development of the Murine Lymphatic System," Cell, Vol. 98, No. 2, 1999, pp. 769-778. doi:10.1016/S0092-8674(00)81511-1

[16] D. Massi, et al., "Tumour Lymphangiogenesis Is a Possi- 
ble Predictor of Sentinel Lymph Node Status in Cutaneous Melanoma: A Case-Control Study," Journal of Clinical Pathology, Vol. 59, No. 2, 2006, pp. 166-173. doi:10.1136/jep.2005.028431

[17] Y. Miyata, et al., "Lymphangiogenesis and Angiogenesis in Bladder Cancer: Prognostic Implications and Regulation by Vascular Endothelial Growth Factors-A, -C, and -D," Clinical Cancer Research, Vol. 12, 2006, pp. 800806. doi:10.1158/1078-0432.CCR-05-1284

[18] H. J. Kahn and A. Marks, "A New Monoclonal Antibody, D2-40, for Detection of Lymphatic Invasion in Primary Tumours," Laboratory Investigation, Vol. 82, 2002, pp. 1255-1257.

[19] Marinho VF, et al., "Lymph Vascular Invasion in Invasive Mammary Carcinomas Identified by the Endothelial Lymphatic Marker D2-40 Is Associated with Other Indicators of Poor Prognosis," BMC Cancer, Vol. 8, 2008, pp. 64-68. doi:10.1186/1471-2407-8-64

[20] S. F. Schoppmann, et al., "Prognostic Value of Lymphangiogenesis and Iymphovascular Invasion in Invasive Breart Cancer," Annals of Surgery, Vol. 240, No. 2, 2004, pp. 306-312. doi:10.1097/01.sla.0000133355.48672.22

[21] X. D. Xie, et al., "Relation of Lymphatic Microvessel Density Detected by Monoclonal Antibody D2-40 with
VEGF-C Expression in Breast Cancer," Zhonghua Zhong Liu Za Zhi, Vol. 30, No. 5, 2008, pp. 356-360.

[22] S. D. Nathanson, "Insights into the Mechanisms of Lymph Node Metastasis," Cancer, Vol. 98, No. 2, 2003, pp. 413423. doi: $10.1002 /$ cncr.11464

[23] G. Gurleyik, et al., "Lymphovascular Invasion, as a Prognostic Marker in Patients with Invasive Breast Cancer," Acta Chirurgica Belgica, Vol. 107, No. 3, 2007, pp. 284287.

[24] R. Jagsi, et al., "Locoregional Recurrence Rates and Prognostic Factors for Failure in Node-Negative Patients Treated with Mastectomy: Implications for Postmastectomy Radiation," International Journal of Radiation Oncology, Biology, Physics, Vol. 62, No. 4, 2005, pp. 10351039. doi:10.1016/j.ijrobp.2004.12.014

[25] B. Kuru, et al., "Prognostic Factors Affecting Survival and Disease-Free Survival in Lymph Nodenegative Breast Carcinomas," Journal of Surgical Oncology, Vol. 83, 2003, pp. 167-172. doi:10.1002/jso. 10264

[26] S. D. Nathanson, et al., "Sentinel Lymph Node Metastasis in Experimental Melanoma: Relationships among Primary Tumour Size, Lymphatic Vessel Diameter and 99mTclabeled Human Serum Albumin Clearance," Annals of Surgical Oncology, Vol. 4, No. 2, 1997, pp. 161168. doi: $10.1007 / \mathrm{BF} 02303800$ 